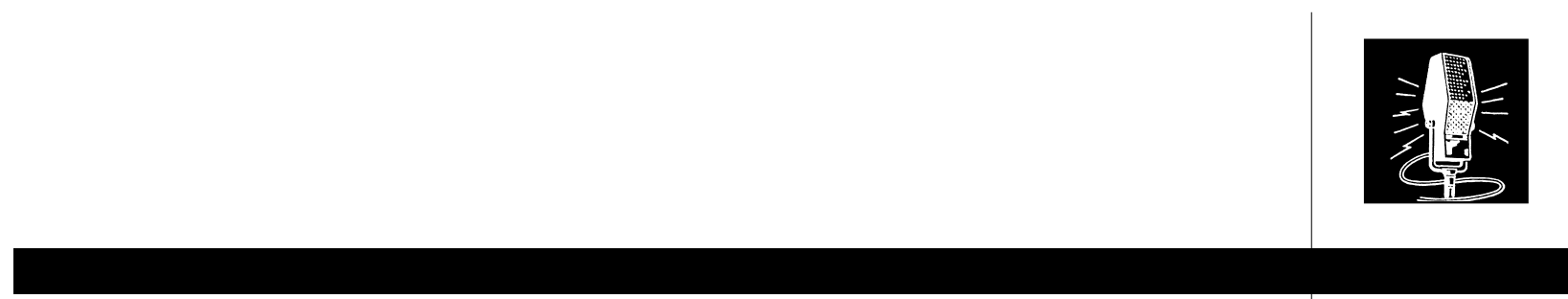

\title{
A Note of Thanks to Our Reviewers
}

The Editors wish to extend their heartfelt thanks to the reviewers of papers published in the first two volumes of the Journal. Critical but fair reviews by experts in the various fields of diabetes research has and will be pivotal to the quality and success of the journal. It is a time consuming exercise in colleague's often busy routine that they volunteer. We are therefore thankful for their time and expertise that have, in no small measures, contributed to two successful initial years of the Journal.
Dale Abrahamson

Leona Aerts

Elif Arioglu

C.J. Bailey

Aydin Barlas

Hanoch Bar-On

T.K. Basu

Geert Jan Biessels

Kersten Buschard

Norman Cameron

Arthur Campfield

Richard Carr

Erol Cerasi

Tova Chajek

Subrata Chakrabarti

Mark E. Cooper

Mary Cotter

Errol D. Crook

N.S. Dhalla
R. Donnelly

Joseph C. Dunbar

Simon J. Dunmore

Shimon Efrat

Joseph Eichberg

George S. Eisenbarth

D.L. Eizirick

Robert B. Elliot

Eva Feldman

Peter Flatt

Josephine Forbes

Robert Frank

Jean Paul Giacobino

Per Henrik Groop

George Grunberger

Barbara Hansen

Leonard C. Harrison

William W. Hay

Bo Helman
Jean Claude Henquin

Douglas N. Henry

Andre Herchuelz

Emilio Herrera

David Hill

Kathleen Holemans

Thomas H. Hostetter

Gokhan S. Hotamisligil

Jorma Ilonen

Bowie Kahle

B. Kahn

Frederik Kaskel

Timothy Kern

George L. King

Iwar Klimes

Gregory Korbutt

Anjaneyulu Kowluru

Anna Krook

D. Langin 
David Lau

Jack Leahy

Edward H. Leiter

Sigurd Lenzen

Åke Lernmark

Zecharia Madar

Errol Marliss

Michelle Marron

Clayton Mathews

Franz Matschinsky

Paolo Meda

Vincent Monnier

David Mott

Ram Nagaraj

Christopher Newgard

Irina Obrosova

Hiroshi Okamoto

Asher Ornoy

Jerry Palmer

Hans Parving

Ammon Peck

Michael Pfeifer

Masimo Pietropaolo
Andreas Plagemann

Bernard Portha

Guiseppe Pugliese

Alexander Rabinovitch

Ralph Rabkin

Arlene Ramsingh

Heimo Riedel

E. Ritz

Ladislas Robert

Jorge Tamarit Rodriguez

Bert Roep

Merja Roivainen

Guillermo Romero

Aldo Rossini

Dvora Rubinger

Ingo Rustenbeck

Stellan Sandler

Robert Schmidt

Fraser Scott

Assia Shisheva

Colin P. Sibley

Bhagirath Singh

Gail E. Sonnenshein
Robert L. Sorenson

Yechezkel Stein

Martin Stevens

Andrew Taylor

Markus Tiedge

Vincenzo Trischitta

Jerrold Turner

Thijen Utkan

Angela M. Valverde

L. Van Gaal

Pnina Vardi

Aaron Vinik

Ken Walder

Michael B. Wheeler

Morris White

John Wiley

Yagahashi, Soroku

Ji-Won Yoon

Mark Yorek

Daniel Ziegler

Juleen Zierath

Ehud Ziv

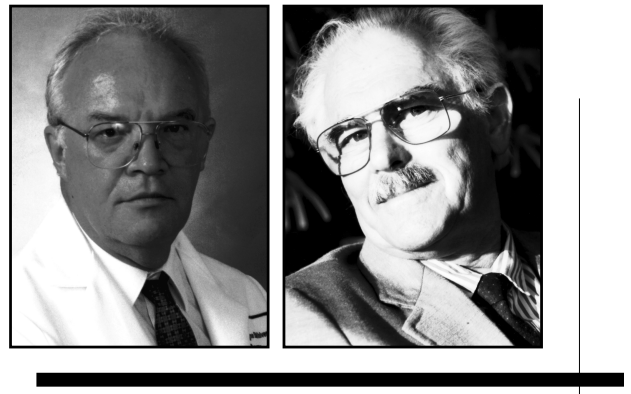

Anders A.F. Sima, Eleazar Shafrir

Editors-in-Chief 


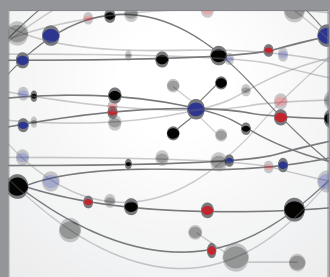

The Scientific World Journal
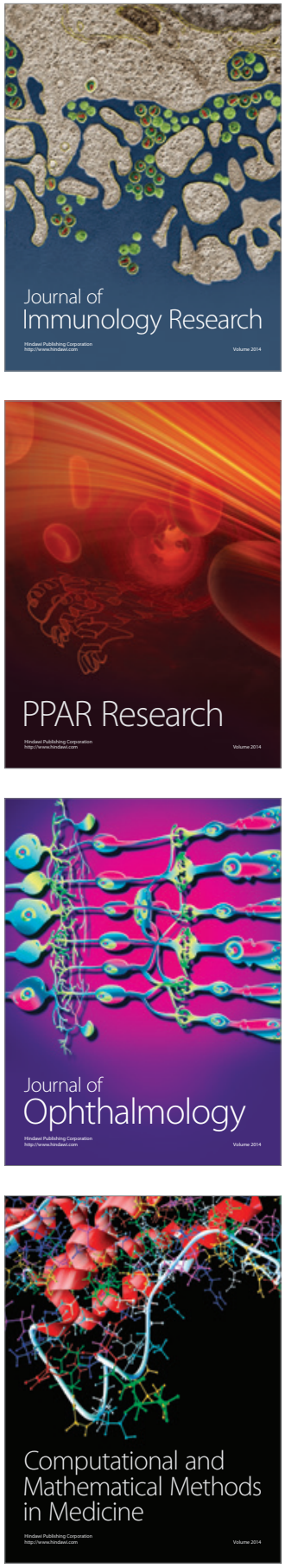

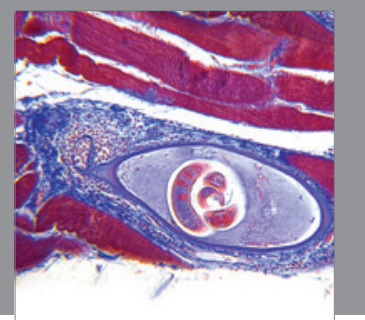

Gastroenterology

Research and Practice
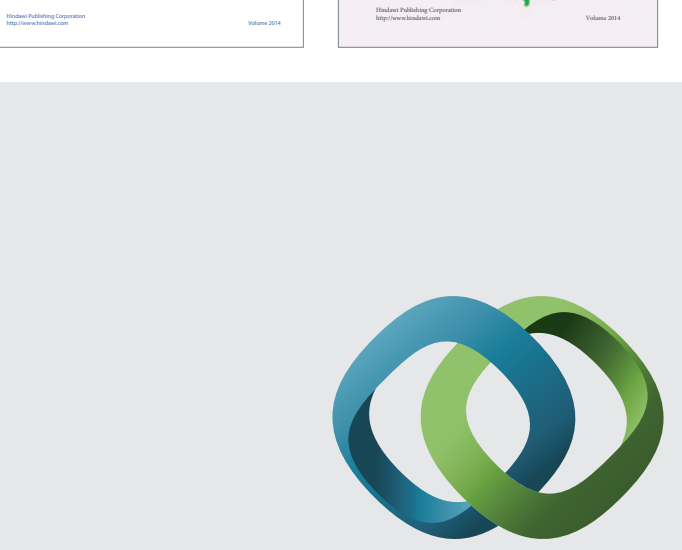

\section{Hindawi}

Submit your manuscripts at

http://www.hindawi.com
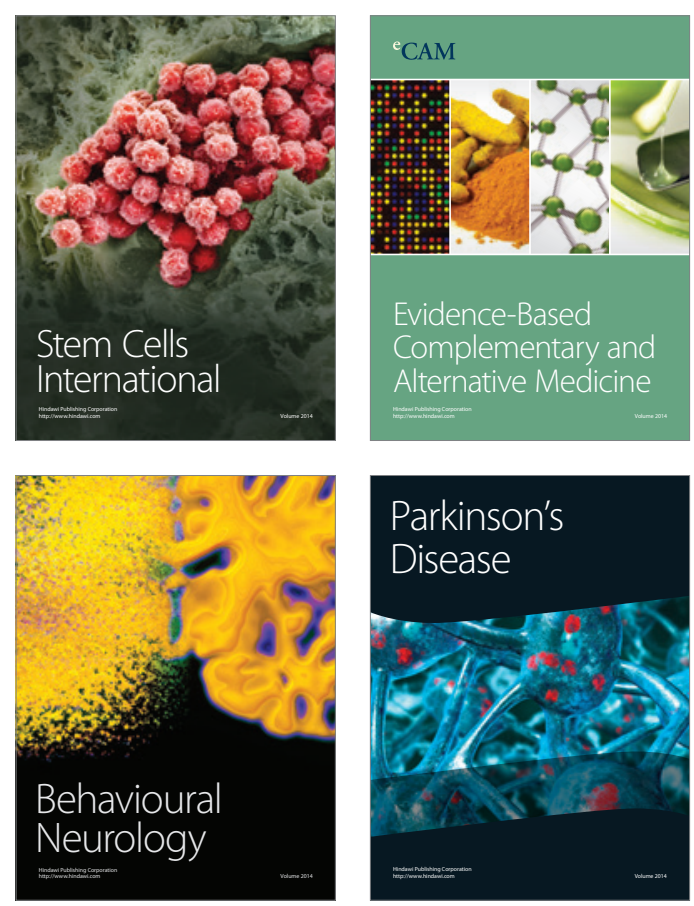

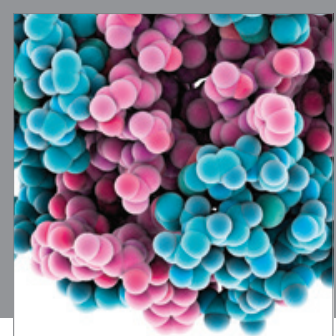

Journal of
Diabetes Research

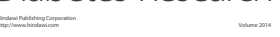

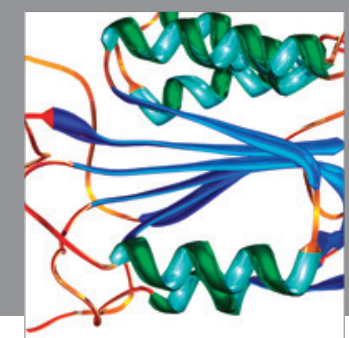

Disease Markers
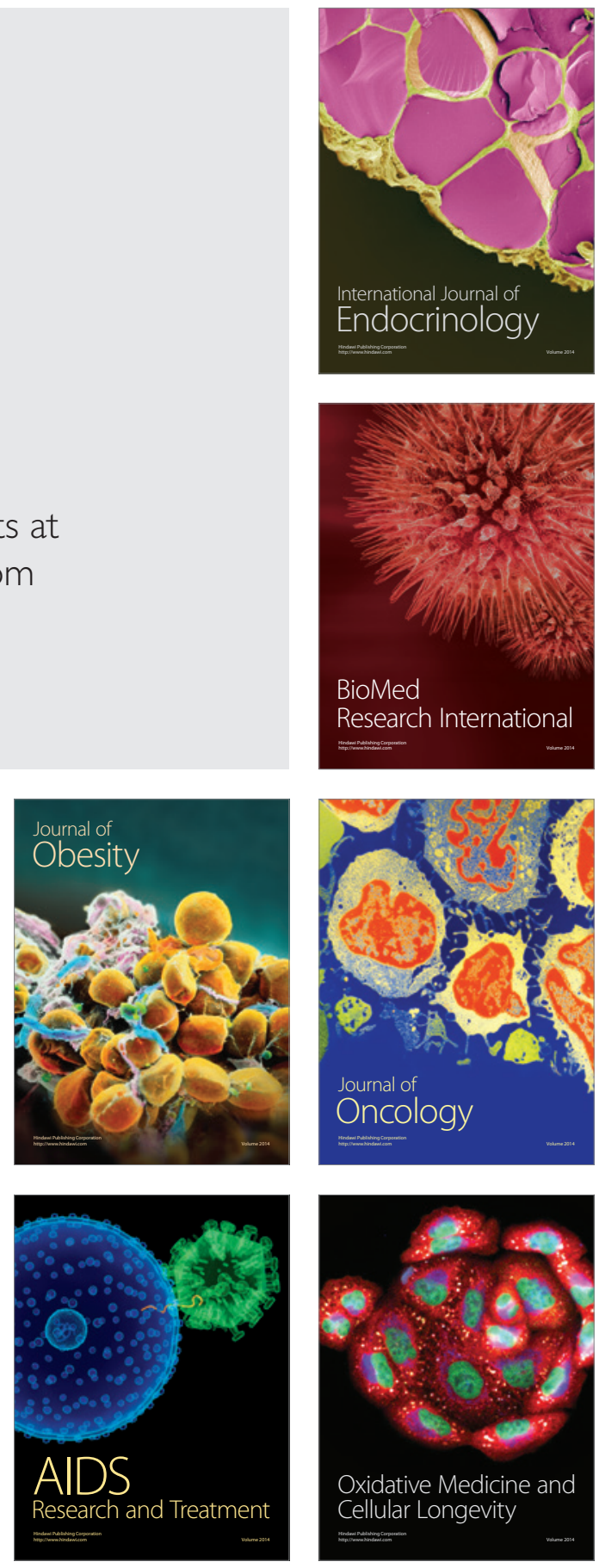